\title{
Pulmonary Embolism with Floating Right Atrial Thrombus Successfully Treated with Streptokinase
}

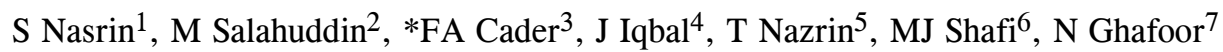 \\ ${ }^{1}$ Dr. Sahela Nasrin, Associate Consultant \& Assistant Professor, Department of Cardiology, Ibrahim Cardiac \\ Hospital \& Research Institute (ICHRI), Dhaka \\ ${ }^{2}$ Dr. Mohammad Salahuddin, Registrar and Specialist, Department of Cardiology, Ibrahim Cardiac Hospital \\ \& Research Institute (ICHRI), Dhaka \\ ${ }^{3}$ Dr. Fathima Aaysha Cader, Assistant Registrar, Department of Cardiology, Ibrahim Cardiac Hospital \\ \& Research Institute (ICHRI), Dhaka \\ ${ }^{4}$ Dr. Md. Jabed Iqbal, Registrar and Specialist, Department of Cardiology, Ibrahim Cardiac Hospital \\ \& Research Institute (ICHRI), Dhaka \\ ${ }^{5}$ Dr. Tahera Nazrin, Associate Consultant \& Assistant Professor, Department of Paediatric Cardiology, \\ Ibrahim Cardiac Hospital \& Research Institute (ICHRI), Dhaka \\ ${ }^{6}$ Dr. Masuma Jannat Shafi, Registrar and Specialist, Department of Cardiology, Ibrahim Cardiac Hospital \\ \& Research Institute (ICHRI), Dhaka \\ ${ }^{7}$ Dr. Nusrat Ghafoor, Associate Consultant, Department of Radiology \& Imaging, Ibrahim Cardiac Hospital \\ \& Research Institute (ICHRI), Dhaka \\ *Corresponding Author
}

Date of submission: 20 January $2015 \quad$ Date of acceptance: 15 April 2015

\begin{abstract}
Massive Pulmonary Embolism (PE) is associated with significant mortality, especially if compounded by haemodynamic instability, right ventricular dysfunction and right atrial thrombus. Thrombolysis can be lifesaving in patients with major embolism and cardiogenic shock, and accelerates the resolution of thrombus. Only three fibrinolytic agents - namely streptokinase, urokinase, and recombinant tissue plasminogen activator (Alteplase) have been approved in the treatment of PE, with studies demonstrating similar safety profiles. We report the case of a 33 year old Bangladeshi female with a history of recent ankle fracture and immobilization, who presented with massive PE, leading to cardiac arrest. Upon rapid resuscitation, urgent echocardiogram revealed right ventricular dysfunction with floating right atrial thrombus, and she was successfully treated with 1.5 million IU of Streptokinase over 2 hours as per accelerated regimen recommended by the European Society of Cardiology (ESC) guidelines, resulting in successful resolution of the right heart thrombus, and significant clinical improvement. Subsequent CT Pulmonary Angiogram confirmed the diagnosis of PE, and she was anticoagulated to a PT/INR of 2.0 to 3.0.
\end{abstract}

Key Words: massive pulmonary embolism, right atrial thrombus, streptokinase

\section{Introduction}

Massive pulmonary embolism (PE) is frequently complicated with hypotension and shock, leading to mortality rates exceeding $50 \% .1,2$ Patients with right ventricular (RV) dysfunction are another subgroup with a guarded prognosis, ${ }^{3}$ as are those with right heart thrombus. ${ }^{4-6}$ These patients in particular, benefit from more intensive therapy with thrombolytic agents in comparison to anticoagulant therapy alone, resulting in reduced mortality to less than 30\%.2,6 Thrombolytic therapy accelerates the resolution of $\mathrm{PE}$, while reducing its recurrence and improving other parameters, such as pulmonary blood flow, lung perfusion, and right ventricular dysfunction. ${ }^{4,7}$ Streptokinase, Urokinase andrecombinant tissue plasminogen activator (Alteplase) are the thrombolytic agents approved for the treatment of PE, with Alteplase being explicitly identified as the agent indicated for acute massive PE. ${ }^{7}$ This case report aims to demonstrate the importance of prompt imaging and intervention, and the superior efficacy of thrombolysis in complicated massive PE. 
Pulmonary Embolism with Floating Right Atrial Thrombus Successfully

\section{Case Report}

A 33 year old normotensive, non-diabetic Bangladeshi female presented with sudden onset severe retrosternal chest pain and two episodes of syncope over four hours. Chest pain was worse on deep inspiration and associated with shortness of breath, orthopnoea and palpitations for 2 days. She had an ankle fracture and was on a cast with plaster immobilization for the preceding month, and admitted to unilateral leg pain and swelling.

On admission, she was cyanosed with gasping respiration; pulse \& BP were non-recordable. She developed asystole soon after, and reverted to sinus rhythm following two minutes of Cardio Pulmonary Resuscitation (CPR). After resuscitation, heart rate was 136 beats/min and blood pressure was $80 / 55 \mathrm{mmHg}$. SpO2 was $90 \%$. Respiratory rate was 35 breaths/min. She was given high flow oxygen, Intravenous (IV) normal saline and dopamine infusion for hypotension. ECG revealed sinus tachycardia (rate 136/min), Right Bundle Branch Block (RBBB) with $\mathrm{S}_{1} \mathrm{Q}_{3} \mathrm{~T}_{3}$ pattern (Figure 1). Bedside echocardiogram revealed floating thrombus in right atrium (RA), dilated RA and RV, impaired RV function, mild Tricuspid Regurgitation \& pulmonary hypertension with normal left ventricular systolic function (Figure 2). Immediate thrombolysis was done with IV Streptokinase 1.5 million units over 2 hours as per accelerated regimen of ESC guidelines, resulting in a subsequently normal ECG (Figure 3). This was followed by subcutaneous Low Molecular Weight Heparin (LMWH) for 5 days \& oral warfarin titrated to a therapeutic PT/INR of 2.0 to 3.0. Subsequent CT Pulmonary Angiogram revealed an approximately $2 \mathrm{~cm}$ filling defect in the descending branch of left pulmonary artery extending up to the lateral \& posterior basal segmental arteries, suggesting thrombus (Figure 4). D-dimer assay was positive. Troponin- I was $1.27 \mathrm{ng} / \mathrm{ml}$ ( high risk 0.11-0.60). Complete blood count revealed neutrophilic leucocytosis. Review echo done 2 days later revealed no thrombus or pulmonary hypertension, normal RA and RV. She was discharged on warfarin $5 \mathrm{mg}$ daily and was asymptomatic with therapeutic PT/INR at follow up. She denied use of the oral contraceptive pill and was advised against its use owing to its potential as a risk factor.

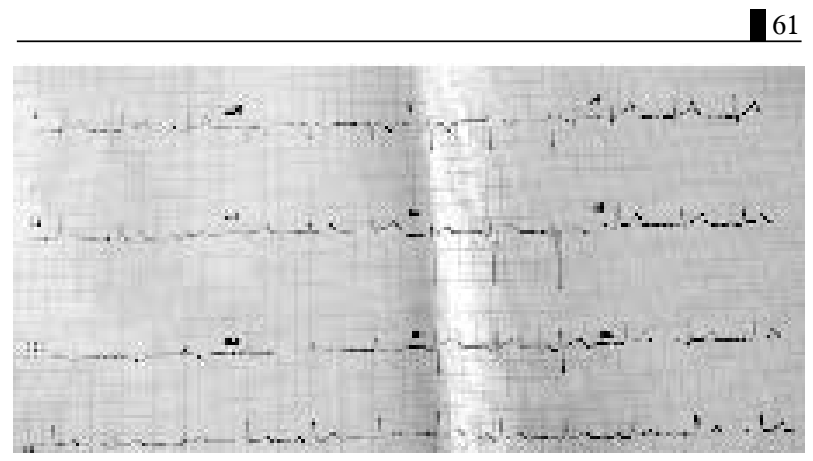

Figure 1. ECG showing sinus tachycardia, RBBB, $\mathrm{S}_{1} \mathrm{Q}_{3} \mathrm{~T}_{3}$ pattern.

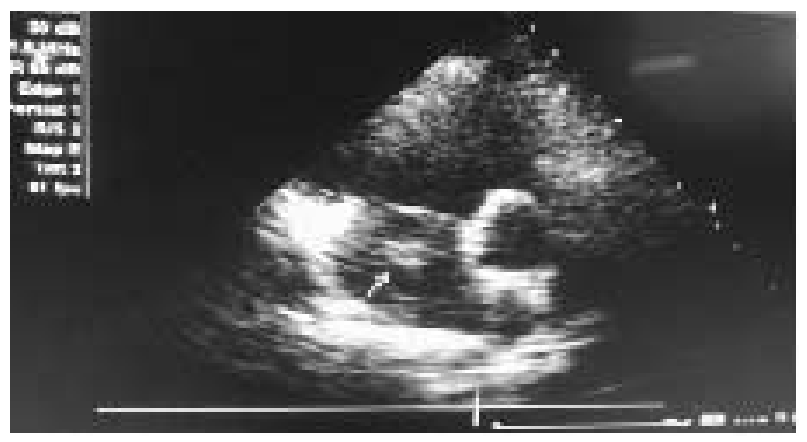

Figure 2: Trans thoracic echocardiogramapical 4 chamber view showing right atrial thrombus.

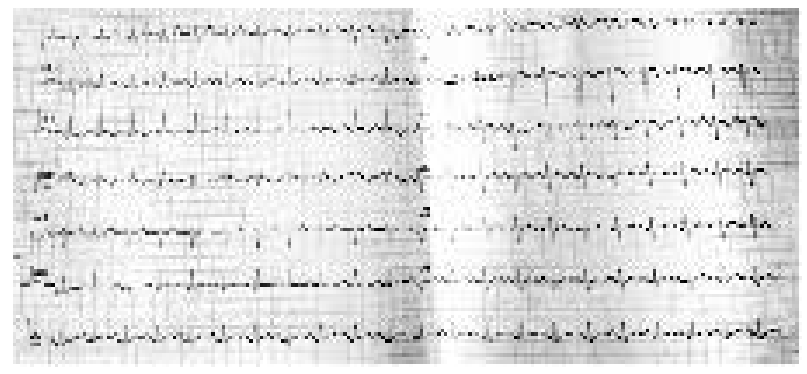

Figure 3. ECG after thrombolysis, showing normal sinus rhythm, rate 72 beats $/ \mathrm{min}$.

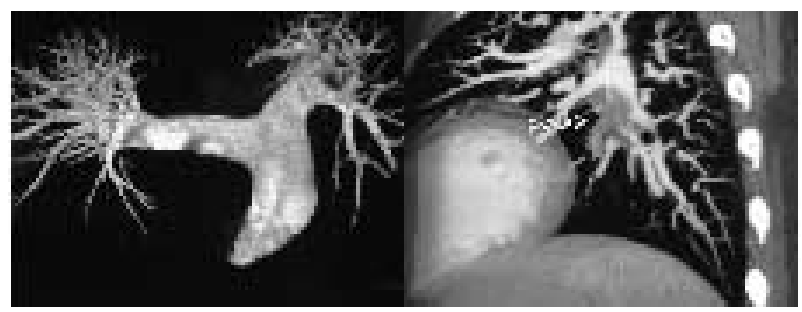

Figure 4. CT Pulmonary Angiogram showing filling defect in the descending branch of Left Pulmonary artery suggesting thrombus 


\section{Discussion}

This case of acute PE presented in cardiorespiratory arrest and is classified as massive PE as per American Heart Association (AHA) definitions. ${ }^{7}$ The AHA defines massive $\mathrm{PE}$ as acute $\mathrm{PE}$ with sustained hypotension (systolic blood pressure $<90 \mathrm{~mm} \mathrm{Hg}$ for at least 15 minutes or requiring inotropic support, not due to a cause other than PE), pulselessness, or persistent profound bradycardia (heart rate $<40 \mathrm{bpm}$ with signs or symptoms of shock). ${ }^{7}$ ESC guidelines also classify suspected acute PE as 'high risk' on the basis of presence of shock or hypotension. ${ }^{4}$

Emergency multidetector CT should be performed in haemodynamically unstable patients, because of its 97\% sensitivity for detecting emboli in the main pulmonary arteries. ${ }^{6,7}$ If unavailable, echocardiography should be performed without delay.6,7 Echocardiographic markers of RV dysfunction, such as RV dilatation (without hypertrophy), paradoxical septal systolic motion, and pulmonary hypertension are independent predictive factors of poor outcome in acute PE. ${ }^{8}$ Echocardiography can also detect right heart thrombi, a marker of worse prognosis, the prevalence of which is $4 \%$ to $18 \%$ in the setting of an acute $\mathrm{PE},{ }^{9}$ and usually found in those more haemodynamically compromised. ${ }^{5,6}$ Free-floating right heart thrombi, are almost exclusively associated with pulmonary embolism. ${ }^{10,11}$

Elevated D dimers and positive cardiac troponin T or I, both of which have a high negative predictive value, can be used for immediate risk stratification. ${ }^{4,7,12}$ A normal D-dimer level renders acute PE or DVT unlikely.

Scoring systems may be adopted for early risk stratification of patients, taking into account the clinical status and risk factors for venous thromboembolism (VTE) such as lower limb fractures, major trauma and surgery. ${ }^{7}$ With a Well's Score of 9, and a Revised Geneva Score of 11, our patient had high clinical probability of PE. ${ }^{13-14}$ She had a Pulmonary Embolism Severity Index (PESI) score of 103, putting her in Class III, with a 30-day moderate mortality risk of 3.2 to 7.1\%.15 According to 2014 ESC guidelines, she had high early mortality risk owing to shock, PESI class III-IV, RV dysfunction on imaging and positive cardiac laboratory markers, thus warranting primary reperfusion. ${ }^{4}$ There is no contraindication to fibrinolysis in cases of cardiac arrest owing to PE, however thrombolysis is discouraged in those with undifferentiated cardiac arrest. ${ }^{7}$ Where patient transport for CT is unsafe, thrombolysis should be considered in case of unequivocal signs of RV overload on bedside echocardiography, and CT performed later. ${ }^{6}$

There are three thrombolytics approved for the treatment of PE by the Food and Drug Administration (FDA): Streptokinase, urokinase and alteplase, with Alteplase being explicitly identified as the agent indicated for massive PE in 2010. ${ }^{7}$ There are no conclusive findings from studies comparing different thrombolytic regimens in acute PE, with most of them demonstrating similar safety profiles. ${ }^{16-18}$ However, short infusion times ( 2 hours or less) are recommended over prolonged infusion times, as they achieve more rapid thrombolysis and probably less bleeding. 7,19

Thrombolytic agents actively promote the hydrolysis of fibrin molecules, resulting in rapid resolution of thromboembolic obstruction, and faster restoration of pulmonary perfusion in the acute stage, with a $30 \%$ to $35 \%$ reduction in total perfusion defect at 24 hours in comparison with heparin alone. 7,19 This leads to a prompt reduction in pulmonary artery pressure and resistance, with a concomitant improvement in RV function, stabilization of respiratory and cardiovascular function, and prevention of PE recurrence. ${ }^{7}$ Major contraindications include haemorrhagic or ischaemic stroke, recent major surgery or trauma or known bleeding risk. ${ }^{4,18}$

Thrombolysis has mortality benefit when compared either to anticoagulation or surgical thromboembolectomy, in cases of right heart thrombus. ${ }^{20}$ Surgicalembolectomy is currently more frequent, but remains limited to patients unsuitable for thrombolysis. Catheter-based embolectomy is reserved for cases in which both thrombolysis and surgical embolectomy is possible. ${ }^{1}$

As patients with acute PE are at risk for recurrent thromboembolism, they should be given long -term anticoagulation. The recommendation for PE secondary to a reversible risk factor is therapy with vitamin $\mathrm{K}$ antagonists for 3 months, titrated to a target INR of 2.0 to 3.0.4,6 Novel oral anticoagulants (NOACs) i.e. dabigatran, rivaroxaban and apixaban are as effective and safe as warfarin for the treatment of venous thromboembolism. , $, 6,7$

Follow up of patients is important, due to implications of long term anticoagulation and the possibility of chronic thromboembolic pulmonary hypertension after an acute PE, the incidence of which is up to $3.8 \%$ two years after the acute event. ${ }^{21}$ 
Pulmonary Embolism with Floating Right Atrial Thrombus Successfully

\section{Conclusion}

Acute massive PE can present with haemodynamic instability and RV dysfunction in predisposed patients. Floating RA thrombus, although rare, is an additional complication. Therefore, prompt diagnosis by confirmation with appropriate imaging techniques and rapid decision to thrombolyse such cases can be life-saving.

\section{Acknowledgements : None}

\section{Conflict of Interest}

The authors declare that they have no conflict of interest.

\section{References}

1. Meneveau N. Therapy for acute high-risk pulmonary embolism: thrombolytic therapy and embolectomy. CurrOpinCardiol. 2010; 25(6): 560-67.

2. Jerjes-Sanchez C, Ramirez-Rivera A, de Lourdes Garcia M, Arriaga-Nava R, Valencia S, Rosado-Buzzo A, et al. Streptokinase \& heparin versus heparin alone in massive pulmonary embolism: a randomized controlled trial. J Thromb Thrombolysis 1995; 2(3): 227-29.

3. Kreit JW. The impact of right ventricular dysfunction on the prognosis and therapy of normotensive patients with pulmonary embolism. Chest 2004; 125(4): 1539-45.

4. Konstantinides SV, Torbicki A, Agnelli G, Danchin N, Fitzmaurice D, Galiè N, et al. 2014 ESC Guidelines on the diagnosis and management of acute pulmonary embolism: The Task Force for the Diagnosis and Management of Acute Pulmonary Embolism of the European Society of Cardiology (ESC). Eur Heart J 2014; 35(43): 3033-73.

5. Torbicki A, Galie N, Covezzoli A, Rossi E, De Rosa M, Goldhaber SZ. Right Heart thrombi in pulmonary embolism: results from the International cooperative pulmonary embolism registry. J Am Coll Cardiol 2003; 41(2): 2245-51.

6. Agnelli G, Becattini C. Acute Pulmonary Embolism. N Engl J Med 2010; 363(3): 266-74.

7. Jaff MR, McMurtry MS, Archer SL, Cushman M, Goldenberg N, Goldhaber SZ, et al. Management of massive and submassive pulmonary embolism, iliofemoral deep vein thrombosis, and chronic thromboembolic pulmonary hypertension: a scientific statement from the American Heart Association.Circulation 2011; 123(16): 1788-830.

8. Grifoni S, Olivotto I, Cecchini P, Pieralli F, Camaiti A, Santoro G, et al. Short-term clinical outcome of patients with acute pulmonary embolism, normal blood pressure, and echocardiographic right ventricular dysfunction. Circulation2000; 101(24): 2817-22.
9. Ferrari E, Benhamou M, Berthier F, Baudouy M. Mobile thrombi of the right heart in pulmonary embolism: delayed disappearance after thrombolytic treatment. Chest 2005; 127(3): 1051-53.

10. X Hou, W Liu, Z Zhang, Z Li. Free-floating right atrial thrombus with acute pulmonary embolism. Thorax 2009; 64(8): 736.

11. Chartier L, Bera J, Delomez M, Asseman P, Beregi JP, Bauchart JJ, et al. Free floating thrombi in the right heart: diagnosis, management, \& prognostic indexes in 38 consecutive patients. Circulation 1999; 99(21): 2779-83.

12. Becattini C, Vedovati MC, Agnelli G. Prognostic value of troponins in acute pulmonary embolism: a meta-analysis. Circulation 2007; 116(4): 427-33.

13. Wells PS, Anderson DR, Rodger M, Ginsberg JS, Kearon $\mathrm{C}$, Gent M, et al. Derivation of a simple clinical model to categorize patients probability of pulmonary embolism: increasing the models utility with the SimpliRED Ddimer. ThrombHaemost 2000; 83(3):416-20.

14. Le Gal G, Righini M, Roy PM, Sanchez O, Aujesky D, Bounameaux H, et al. Prediction of pulmonary embolism in the emergency department: the revised Geneva score. Ann Intern Med 2006; 144(3): 165-71.

15. Righini, M, Roy, PM, Meyer G, Verschuren F, Aujesky D, Le Gal G. The Simplified Pulmonary Embolism Severity Index (PESI): validation of a clinical prognostic model for pulmonary embolism. Journal of Thrombosis and Haemostasis 2011; 9(10): 2115-17.

16. Urokinase-streptokinase embolism trial. Phase 2 results. A cooperative study. JAMA 1974; 229(12): 1606-13.

17. Godlhaber SZ, Kessler CM, Heit J, Warkis J, Sharma GV, Dawley D, et al. Randomized control trial of recombinant tissue plasminogen activator versus urokinase in acute pulmonary embolism. Lancet. 1988; 2(8606): 293-98.

18. Meyer G, Sors H, Charbonnier B, Kasper W, Bassand JP, Kerr IM, et al. Effects of intravenous urokinase versus alteplase on total pulmonary resistance in acute massive pulmonary embolism: an European multicentr double blinded trial. The European Cooperative Study Group for pulmonary embolism. J Am Coll Cardiol 1992; 19(2): 239-45.

19. Kearon C, Kahn SR, Agnelli G, Goldhaber S, Raskob GE, Comerota AJ. Antithrombotic therapy for venous thromboembolic disease: American College of Chest Physicians Evidence-Based Clinical Practice Guidelines (8th Edition). Chest 2008; 133(6 Suppl): S454-545.

20. Rose PS, Punjabi NM, Pearse DB. Treatment of right heart thromboemboli. Chest 2002; 121(3): 806-14.

21. Pengo V, Lensing AW, Prins MH, Marchiori A, Davidson BL, Tiozzo F, et al. Incidence of chronic thromboembolic pulmonary hypertension after pulmonary embolism.N Engl J Med. 2004; 350(22): 2257-64. 